\title{
MiR-139-5p alleviates neural cell apoptosis induced by spinal cord injury through targeting TRAF3
}

\author{
Ziying Zhang ${ }^{\varpi}$, Lifang Shen and Yingying Yan \\ Department of Rehabilitation Medicine, The Fifth Affiliated Hospital of Zhengzhou University, Z, Zhengzhou, Henan Province, 450052, PR China
}

Spinal cord injury $(\mathrm{SCl})$ is a neurological trauma that causes loss of locomotor function and sensory deficit. Previous studies showed that miRNAs play a crucial role in $\mathrm{SCl}$. This study further evaluated the potential role of miR-139-5p in the neural cell apoptosis after $\mathrm{SCl}$ in rats. A rat $\mathrm{SCl}$ model was successfully established and miR139-5p expression level in $\mathrm{SCl}$ rats was down-regulated compared to the sham group (sham operation group) determined by qRT-PCR. MiR-139-5p overexpression via administration with miR-139-5p agomir improved locomotor functional recovery, attenuated allodynia and hyperalgesia and alleviated neural cell apoptosis in $\mathrm{SCl}$ rats. In addition, TRAF3 (TNF receptor-associated factor 3) was identified to be a target of miR-139-5p by searching the proposed target genes in TargetScan 7.1 database. Co-transfection of miR-139-5p agomir and adenovirus of TRAF3 plasmids significantly improved functional recovery and alleviated neural cell apoptosis. Therefore, TRAF3 mediated the anti-apoptosis effect of miR-139-5p in SCl rats and miR-139-5p could be a promising candidate for $\mathrm{SCl}$ therapy by alleviating neural cell apoptosis through targeting TRAF3.

Key words: miR-139-5p, spinal cord injury, apoptosis, TRAF3

Received: 04 March, 2020; revised: 22 May, 2020; accepted: 22 May, 2020; available on-line: 27 August, 2020

囚e-mail: ZiyingZhang159@163.com

Abbreviations: $\mathrm{SCl}$, Spinal cord injury; miRNAs, MicroRNAs; SD, Sprague-Dawley; NC, Negative control; Basso, BBB Assay, Beattie, and Bresnahan Assay; qRT-PCR, Real-time polymerase chain reaction

\section{INTRODUCTION}

Spinal cord injury (SCI) is a neurological trauma which causes loss of locomotor function and sensation deficit and involved in high disability and mortality (Varma et al., 2013; Silva et al., 2014; Abdullahi et al., 2016; Kadoya et al., 2016; Ahuja et al., 2017; Bhimani et al., 2017; Diogo et al., 2019). Although various strategies such as hormonal therapy and cell transplantation have been adopted in SCI treatment, it is still difficult to cure neurological defects for improving functional recovery of SCI patients (Nakamura \& Okano, 2013; Ludwig et al., 2017). Previous research suggested that the primary injuries are closely related to mechanical insult such as traffic accidents, overburden and so on. Based on the primary injuries, the secondary injuries including neural cell apoptosis, excessive inflammation, vascular infarction and excitotoxicity were usually observed and prolonged and complicated the clinical therapies after SCI (Oyinbo, 2011; Anwar et al., 2016). Moreover, previous study demonstrated that prevention of the secondary in- juries contributed to the recovery of SCI rats (Anwar et al., 2016). Therefore, more effort in the improvement of secondary injuries may be a promising strategy for SCI therapy.

MicroRNAs (miRNAs) are identified as a class of 18-22 nucleotides non-coding short RNAs which could specifically complement and bind to the 3'-UTR of the target gene, and participate in the regulation of cell cycle, growth and apoptosis, as well as the pathogenesis of various diseases (Croce \& Calin, 2005; Hwang \& Mendell, 2007; Chivukula \& Mendell, 2008). MiRNAs have been shown to play a crucial role in SCI. miR-137 inhibits neuronal cell apoptosis after SCI by targeting MK2 (Gao et al., 2018). miR-221 alleviated neuronal apoptosis induced by spinal cord ischemia-reperfusion via regulating the expression of TNF- $\alpha$ (tumor necrosis factor- $\alpha$ ) induced protein 2 (Zhao et al., 2018). miR-9 down-regulated the expression of monocyte chemotactic proteininduced protein 1 (MCPIP1) and led to the suppression of neuronal cell apoptosis in acute spinal cord injury $(\mathrm{Xu}$ et al., 2016). miR-136 protected neurocytes against apoptosis by targeting tissue inhibitor of metalloproteinases-3 in spinal cord ischemia injury model (Jin et al., 2017).

Previouse studies showed that miR-139-5p is downregulated in neurons, which reduced ischemia and hypoxia-induced neuronal apoptosis through the inhibition of human growth and transformation-dependent protein (HGTD-P) expression (Qu et al., 2014). However, whether miR-139-5p has an effect on the SCI-induced neuronal apoptosis has not been reported. Thus, our study aimed to explore the potential role of miR-139-5p in the neural cell apoptosis after SCI in rats. We found that miR-139-5p reduced SCI-induced neural cell apoptosis through targeting TNF receptor-associated factor 3 (TRAF3). Therefore, miR-139-5p could be a promising candidate for SCI therapy by alleviating neural cell apoptosis through targeting TRAF3.

\section{MATERIALS AND METHODS}

Animals. Adult male Sprague-Dawley (SD) rats (Yison Bio, Shanghai, China) weighing 180-220 g (6-8 weeks) were fed in plastic chambers with a $12 \mathrm{~h}$ light/ dark cycle from 8:00 am in the morning to 8:00 pm in the evening. All rats were raised in the same temperature-controlled room of $\left(25^{\circ} \mathrm{C}\right)$ with adequate water and food. Rats were euthanized 4 weeks after SCI, and then their brains were removed for the following experiments. Then, the expression of TRAF3 was detected using, Western blotting and immunohistochemistry. Meanwhile, microRNA expression was detected by real-time PCR. The experimental procedures and animal care were performed and approved by the Institutional Animal Care 
and Use Committee of the fifth affiliated hospital of Zhengzhou University (approval NO.2017Z086).

Establishment of SCI model. Establishment of the SCI model in rats was performed as described before (Liu et al., 2015; Liu et al., 2004). Rats were randomly divided into two groups (sham operation group (sham group) ( $n=6)$ and SCI $(n=6)$ ). The spinal cord injuries were then produced by moderate contusion using the Allen's weight drop apparatus at thoracic vertebra level 8. The rats were anesthetized with pentobarbital $(50 \mathrm{mg} /$ $\mathrm{kg}$, intraperitoneally). After anesthetization, a laminectomy was carried out at thoracic vertebra level 8 (T8) to expose the spinal cord. The spinal cord was then moderately contused by a modified Allen's weight-drop apparatus $(2.5 \mathrm{~mm}$ in diameter, $10 \mathrm{~g}$ weight dropped from a height of $12.5 \mathrm{~mm}$ ) to produce SCI. Sham animals received a dorsal laminectomy, then underwent a T8 laminectomy only, without weight drop injury. When rats developed bilateral hindlimb twitching and tail flicking, the successful establishment of SCI model was confirmed.

Administration of miR-139-5p agomir and adenovirus of TRAF3 plasmids. For intrathecal injection experiments, drugs were delivered via intrathecal catheter. Then, MiR-139-5p agomir (20 nM in a total volume of $5 \mu \mathrm{l}$ ), negative control (NC) or adenovirus of TRAF3 plasmids $(109 \mathrm{TU} / \mathrm{mL}$ in a total volume of $10 \mu \mathrm{l})$ were given after $1 \mathrm{~h}$ of SCI on the first day for 10 consecutive days. All the rats were fasted for $12 \mathrm{~h}$ from the food and fasted $4 \mathrm{~h}$ from water before operation. The rats were kept warm during the operative process. Rats were anesthetized with intramuscular 10\% Chloral Hydrate (3.5 mg/kg) solution (Yaksh et al., 1976; Hama et al., 2009; Jergova et al., 2016). MiR-139-5p agomir and NC agomir were synthesized by GenePharma (Suzhou, China) and adenovirus of TRAF3 plasmids was purchased from Genecreate (Wuhan, China). The oligonucleotide sequence of miR-139-5p agomir was described as before (5' GACCUCUGUGCACGUGACAUCU 3') (Qu et al., 2014). Rats were then euthanized at indicated time and samples were harvested for the further analysis.

Basso, Beattie and Bresnahan (BBB) Assay. We determined the locomotor function of the rats in different groups once a week as described before (Basso et al., 1995). Three independent observers evaluated the locomotor function according to $\mathrm{BBB}$ locomotor rating scale. It was performed by scoring ranging from 0 (indicated no observable movement) to 21 (indicated normal movement) in each group and the average was determined as the final score. All BBB Scales of SCI Group are 0 . Moving slowing was often found in Sham group, but this phenomenon returned to normal at the 4 th or 5 th day after operation. BBB score of Sham group was significantly higher than SCI group $(p<0.05)$. The movement included hindlimb movement, joint movement, gait stability, and the position of the claw and tail. Observations were performed at 8-12 pm daily, and 5 min were required to observe each rat along with bladder emptying.

Paw withdrawal threshold and thermal withdrawal latency assessment. Mechanical allodynia was determined by examining the paw withdrawal threshold (PWT) with electronic Von Frey filaments (North Coast Medical, Gilroy, CA, USA). Thermal hyperalgesia was assessed by testing the thermal withdrawal latency (PWL) with a BME-410C thermal pain stimulator (Chinese Academy of Medical Sciences, Beijing, China). The device consists of a box placed upon 2 separate heating plates. Temperatures of heating plates increase at a rate of $5^{\circ} \mathrm{C}$ per second and are controlled by temperature controllers. When perceiving the thermal stimulus, the animal typically escapes to the opposite nonheated plate (Tabo et al., 1998; Choi et al., 2012). Each measurement was repeated 3 times every 15 minutes and an average was taken as threshold. This evaluation was conducted once a week after SCI surgery.

Hematoxylin-Eosin (HE) staining. Neuronal damage was assessed using of $\mathrm{HE}$ staining. At $72 \mathrm{~h}$ postinjury, the spinal cord tissue sections were harvested and fixed with 4\% PFA (Paraformaldehyde). After $12 \mathrm{~h}$, coronal spinal cord slices $(4 \mu \mathrm{m})$ located at $400 \mu \mathrm{m}$ rostral to the injury epicenter were selected for analyses. The sections were stained with $0.5 \%$ cresyl violet acetate for $15 \mathrm{~min}$ and the staining were counted via Image-Pro Plus 6.0 software.

TUNEL (terminal dexynucleotidyl transferase (TdT)-mediated dUTP nick end labeling) staining. TUNEL assay kit (abcam, ab66110, USA) was used to determine apoptosis, according to the manufacturers' directions. First, spinal cord fixed sections were heated to $60^{\circ} \mathrm{C}$ for $1 \mathrm{~h}$. And then, according to the standard guidelines, the fixed tissue were dewaxed and rehydrated. The sections were incubated with an appropriate concentration of working liquid protease $\mathrm{K}$ for $15 \mathrm{~min}$, at room temperature. After washing with PBS, tissue sections were covered with TUNEL reaction liquid at $37^{\circ} \mathrm{C}$ for $1 \mathrm{~h}$ followed with DAPI. The cells were recorded in 5 random fields under a photomicroscope $(200 \times$ magnification; IX71 Olympus). Numbers of TUNEL positive cells (with green fluorescent) were counted via ImagePro Plus 6.0.

Dual-luciferase reporter assay. HEK-293T cells were purchased from $\mathrm{BeNa}$ Culture Collection Company (Kunshan, Jiangsu, China). HEK-293T cells were cotransfected with $1 \mu \mathrm{g}$ of the Renilla control luciferase vector (Promega, Madison, WI, USA) and $1 \mu \mathrm{g}$ of the firefly-tagged pGL3 promoter luciferase vector (Promega) containing wild-type or mutant TRAF3 3'-UTR by using FuGENE in each well (Roche Molecular Biochemicals). Additionally, each well was co-transfected with the miR-139-5p mimics or NC mimics (100 $\mathrm{nM})$. Transfected cells were cultured for another 48 hour and then lysed for luciferase activity detection with a dual luciferase assay system (Promega). Results were shown as the relative value of firefly/Renilla.

Cell culture. HEK293T or VSC4.1 cells were seeded at a density of $5 \times 10^{4} / \mathrm{cm}^{2}$ in collagen coated 6-well plates with DMEM/Ham's F12 medium supplemented with $10 \%$ fetal bovine serum and incubated at $37^{\circ} \mathrm{C}$ under a humidified atmosphere of $5 \% \mathrm{CO}_{2}$.

Real-time polymerase chain reaction (qRT-PCR). Samples of injured spinal cords were collected at 28 days after SCI surgery and total RNA were extracted using RNA simple total RNA kit (Tiangen biotech, Beijing, China) according to the manufacturer's instructions. The concentration and quality of total RNA were determined by spectrophotometric determination at 260/280 $\mathrm{nm}$, and equal amounts of RNA from each sample were reverse-transcribed to cDNA for quantitative RT-PCR with the QuantMir RT KIT (System Biosciences, Mountain View, CA, USA). Real-time PCR was then carried out and the miR-139-5p-specific forward primer sequence was designed based on the miRNA sequence. Results were normalized to rat U6 snRNA. The cDNA was synthesized from $1 \mu \mathrm{g}$ of total RNA in a $25 \mu \mathrm{l}$ volume. Real time PCR was carried out with the reagents of a Sybr Green I Mix (Takara, Japan) in a $20 \mu$ l reaction volume $(10 \mu \mathrm{l}$ Sybr Green I Mix, $200 \mathrm{mM}$ forward and reverse primers, $1 \mu \mathrm{l}$ cDNA template) on an MJ Opti- 
con Monitor chromo4 instrument (Bio-Rad, USA) using the following protocol: $95^{\circ} \mathrm{C}$ for $20 \mathrm{~s}$; 40 cycles of $95^{\circ} \mathrm{C}$ for $10 \mathrm{~s}, 60^{\circ} \mathrm{C}$ for $20 \mathrm{~s}, 70^{\circ} \mathrm{C}$ for $1 \mathrm{~s}$. Forward primer for miR-139-5p: 5'GCGGCGGTCTACAGTGCACGTG3'. Reverse primer for miR-139-5p: 5'ATCCAGTGCAGGGTCCGAGG3' U6 forward primer, 5-ACGCA AATTCGTGAAGCGT'T-3, reverse primer, Uni-miR qPCR primer.

Western blotting. The protein samples of injured spinal cords were extracted with RIPA (Radio-Immunoprecipitation Assay) lysis buffer. The equal amount of proteins from each sample were separated by SDS-PAGE (sodium dodecyl sulfate-polyacrylamide gel electrophoresis) and then transferred onto nitrocellulose membranes (Millipore). After the membranes blocked with $5 \%$ skim milk in PBS, the membranes were incubated with primary antibodies for overnight at $4^{\circ} \mathrm{C}$. Primary antibodies in this study including TRAF3 (SAB1406552, 1:500), Bcl-2 (PRS3335, 1:1000), Bax (B3428, 1:500), Cleaved-caspase-3 (C9598, 1:500), and GAPDH (G9295, 1:2000) were purchased from Sigma-Aldrich (St. Louis, MO, USA). After washing with PBST for four times (5 minutes for each time), the membranes were incubated with the HRP-conjugated secondary antibodies (Beyotime biotechnology). Then bands were visualized using a ECL kit (Pierce, USA) and a Tanon analysis system (Biorad, USA) and quantified by ImageJ (the band intensity of total proteins was normalized to loading control, GAPDH).

Measurement of caspase-3 activity. The tissue samples were obtained after SCI. The injury spinal cord tissues were obtained and processed to tissue homogenate. After centrifugation at $1000 \times g$ at $4^{\circ} \mathrm{C}$ for $10 \mathrm{~min}$, each supernatant was transferred to a fresh $1.5-\mathrm{ml}$ centrifuge tube on ice, and protein concentration was determined with a BCA assay kit (Thermo Scientific, USA). Cas- pase-3 activity was measured spectrophotometrically by using caspase-3 activity assay kit (G015, Nanjing Jiancheng, Nanjing) in the supernatant according to the corresponding kit instructions.

Statistical analysis. All results in the present study were presented as the mean \pm standard deviation (SD). Data were analyzed using either a one-way ANOVA with Bonferroni's correction for comparison of multiple groups or an independent Student's t-test for comparison of two groups. The software GraphPad Prism 6.0 was used for data analysis. $p<0.05$ was considered as statistical significant.

\section{RESULTS}

\section{MiR-139-5p was down-regulated in $\mathrm{SCI}$ rats}

The SD rat SCI model was established by moderate contusion of spinal cord. BBB scores at $0,7,14,21$ and 28 days after injury were examined to assess locomotor function recovery. The BBB scores in the SCI group were significantly decreased compared with the sham group (sham operation group) after SCI and gradually elevated throughout the experimental process (Fig. 1A). The expression of miR-139-5p was detected by qRTPCR and the results showed that miR-139-5p level was significantly decreased in SCI group (Fig. 1B). Mechanical allodynia and thermal hyperalgesia presented as PWT and PWL were then further examined. Our data showed that PWT and PWL were remarkably decreased in the SCI group at different time point during the completely experimental period. This suggested that SCI caused significant decrease in mechanical allodynia and thermal hyperalgesia (Fig. 1C and 1D). HE staining showed that the Sham group has no spinal cord loss and syringo-

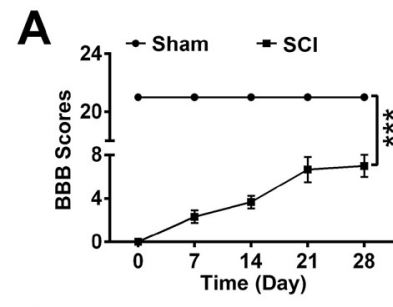

B
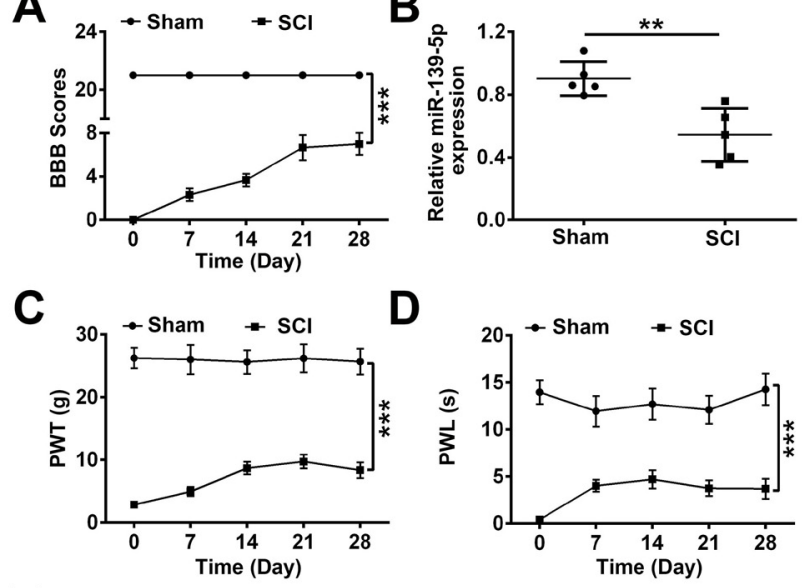

E
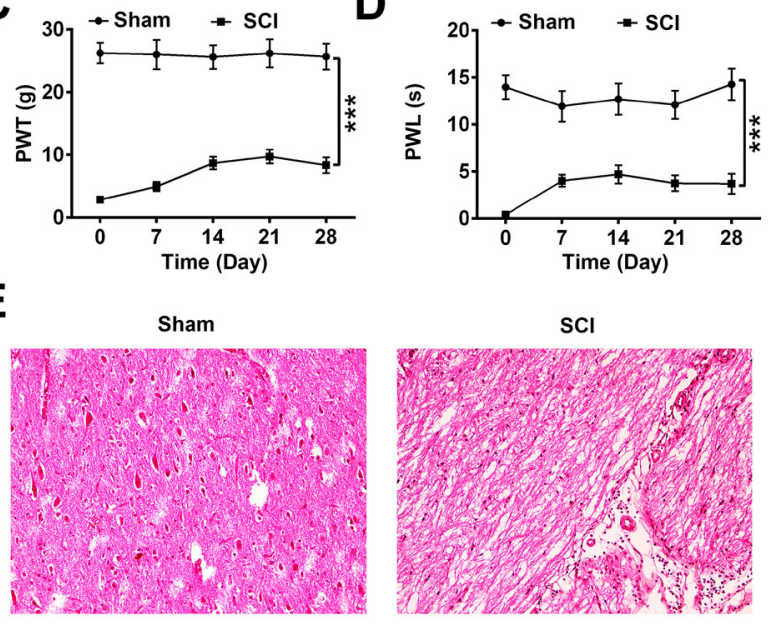

Figure 1. Increased expression of miR-139-5p in SCl rats.

(A) The BBB scores at 0, 7, 14, 21 and 28 days after SCI. (B) Decreased miR-139-5p expression in SCI rats. (C) PWT were detected. (D) PWL were tested at $0,7,14,21$ and 28 days after $\mathrm{SCl}$. $\mathrm{N}=5,{ }^{* *} p<0.01$ or ${ }^{* * *} p<0.001$ refers to the comparison between sham group and $\mathrm{SCl}$ group. (E) HE staining of spinal cord sections located at $400 \mu \mathrm{m}$ rostral to the injury epicenter. $\mathrm{N}=5$ in each group. Scale bar $=200 \mu \mathrm{m}$. 

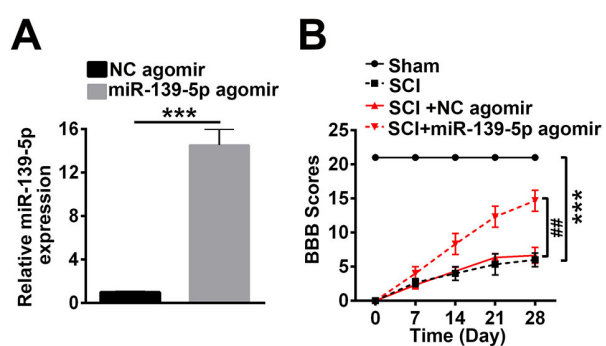

D
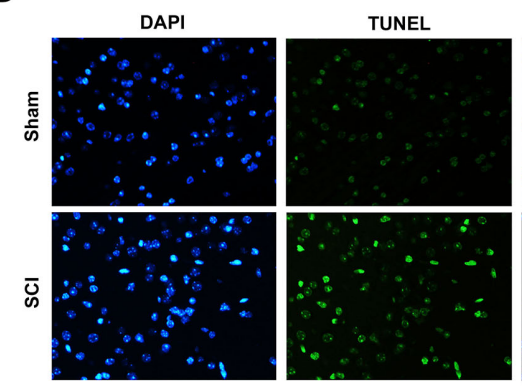

$\mathbf{F}$

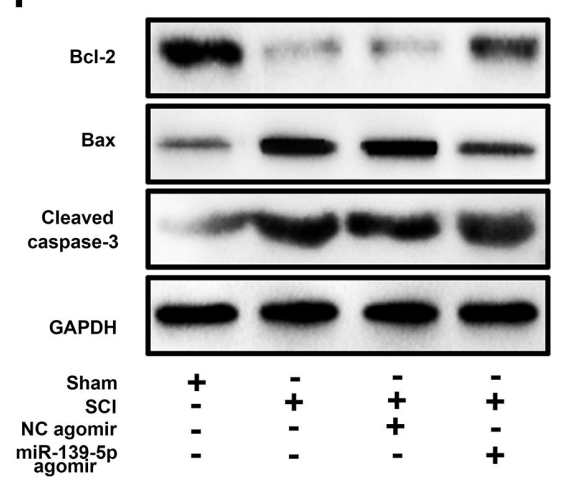

C
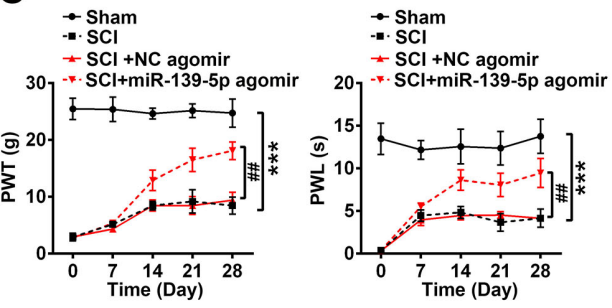

E
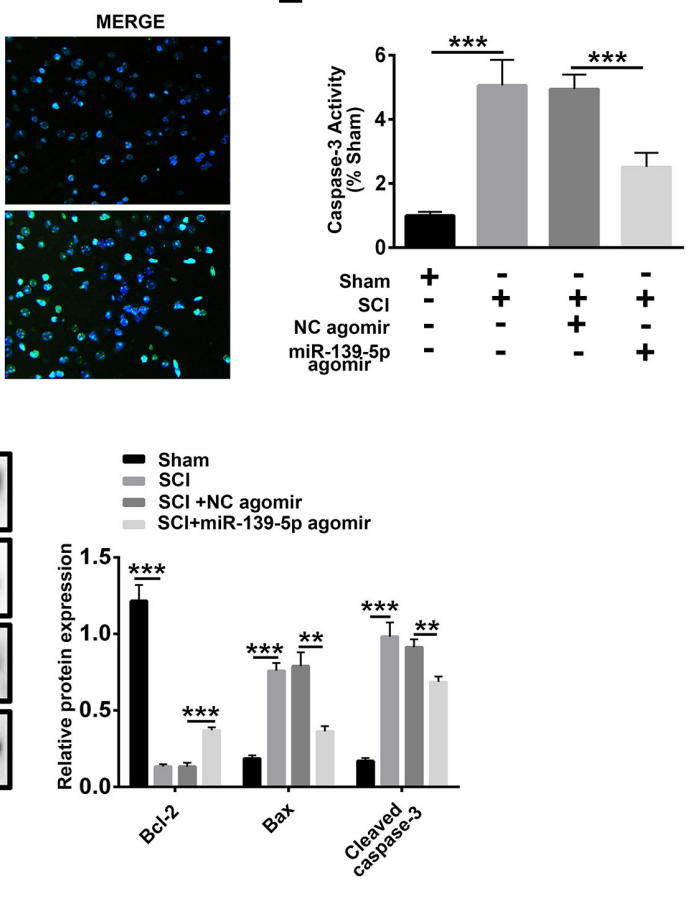

Figure 2. MiR-139-5p overexpression improved locomotor functional recovery, attenuated allodynia and hyperalgesia and alleviated neural cell apoptosis in $\mathrm{SCl}$ rats.

(A) Up-regulated miR-139-5p expression by miR-139-5p agomir. (B) The BBB scores at indicated time. (C) PWT and PWL were detected at $0,7,14,21$ and 28 days after SCl. (D) Elevation of miR-139-5p significantly suppressed apoptosis in SCI rat models as detected by TUNEL staining. All experiments were repeated in triplicate and all data were shown as mean \pm S.D. ( $N=6$, each group). (E) Caspase-3 activity in various groups. (F) Bcl-2, Bax and Cleaved caspase-3 level. $\mathrm{N}=6,{ }^{* *} p<0.01$ and ${ }^{* * *} p<0.001$ refer to the group compared with the sham group. ${ }^{\# p} p<0.01$ refers to the group compared with the group of $\mathrm{SCl}+\mathrm{NC}$ agomir.

mymal formation, while the HE staining of SCI group was lighter and tissue structure was disordered compared with that of Sham rats (Fig. 1E). These results demonstrated that the rat SCI model was successfully established and miR-139-5p was down-regulated in SCI rats.

\section{MiR-139-5p overexpression alleviates neural cell apoptosis after $\mathrm{SCl}$}

To clarify the effect of miR-139-5p on spinal cord injuries, SCI rats were treated with miR-139-5p agomir or NC agomir for miR-139-5p overexpression by local intrathecal injection into the cord. qRT-PCR results indicated high efficacy of miR-139-5p overexpression by agomir injection (Fig. 2A). The BBB scores were significantly increased in miR-139-5p agomir group. This result suggested up-regulation of miR-139-5p significantly improved locomotor functional recovery (Fig. 2B). Moreover, PWT and PWL were also significantly elevated suggesting that miR-139-5p agomir overexpression attenuated allodynia (pain due to a stimulus that does not usually provoke pain) and hyperalgesia (increased pain from a stimulus that usu- ally provokes pain) caused by SCI in rats (Fig. 2C). The effect of miR-139-5p on neural cell apoptosis was then examined. Caspase- 3 activity was dramatically increased due to spinal cord injuries and then profoundly decreased after miR-139-5p agomir treatment (Fig. $2 \mathrm{D}-\mathrm{E})$. In addition, the expression of $\mathrm{Bcl}-2$ was markedly decreased in the SCI group whereas increased in miR-139-5p agomir group, and the expression of Bax and caspase-3 showed consistent results (Fig. 2F). These data suggested that overexpression of miR-139$5 \mathrm{p}$ improved spinal cord injuries by alleviating neural cell apoptosis.

\section{TRAF3 is a target of miR-139-5p}

105 potential target genes of miR-139-5p were examined by searching of TargetScan 7.1 database and filtered through the DAVID website. In this study, we specifically chose TRAF3 as a target of miR-139$5 p$ based on two principles. The protein is related to apoptosis and has not been reported. In addition, TRAF3 was selected by literature review. The complementary sequence of miR-139-5p was observed in 
A

Position 4872-4879 of TRAF3 3'UTR

TRAF3 WT 5, ..AAUAAAAUUUGUAAGACUGUAGA.

miR-139-5p 3' GACCUCUGUGCACGUGACAUCU

TRAF3 MUT 5' ...AAUAAAAUUUGUAAGUGACAUCA.

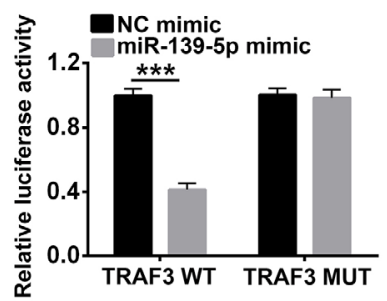

B

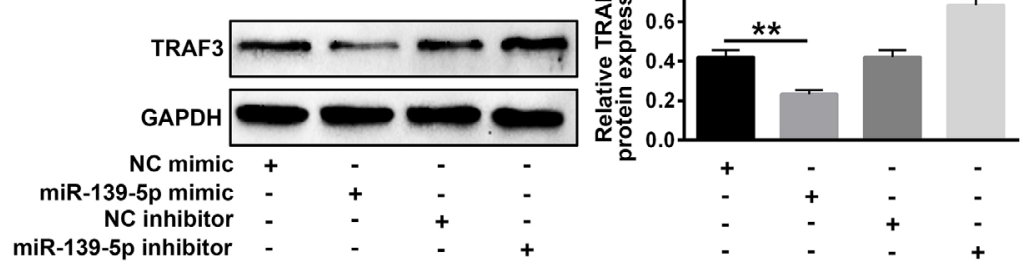

Figure 3. TRAF3 is a target of miR-139-5p.

(A) The putative binding site of miR-139-5p and TRAF3 (left); Luciferase activity of TRAF3 with wild-type or mutated 3'-UTR in HEK-293T cells (right). (B) The TRAF3 protein expression in VSC4.1 cells with various treatments. ${ }^{* *} p<0.01$ and ${ }^{* * *} p<0.001$ refers to the comparison between groups transfected with miR-139-5p control and mimic.

3'-UTR of TRAF3 mRNA. Our data showed that luciferase activity of TRAF3 of wild-type (W'T) 3'-UTR was significantly decreased upon overexpression of miR-139-5p whereas mutant 3'-UTR of TRAF3 was not influenced (Fig. 3A). Furthermore, the TRAF3 protein expression was detected by Western blotting in VSC4.1 cells treated with NC mimic, miR-139-5p mimic, NC inhibitor or miR-139-5p inhibitor. These results showed that overexpression of miR-139-5p decreased the expression of TRAF3 while miR-139-5p knockdown up-regulated the expression of TRAF3
(Fig. 3B). These data demonstrated that TRAF3 is a target of miR-139-5p.

\section{MiR-139-5p alleviates neural cell apoptosis by targeting} TRAF3

Since TRAF3 was proved to be the target of miR139-5p, SCI rats were administrated with miR-139-5p agomir and adenovirus of TRAF3 plasmids to check whether TRAF3 mediated the anti-apoptosis effect of miR-139-5p in SCI rats. qRT-PCR results suggested high efficacy of TRAF3 overexpression (Fig. 4A). The BBB
A

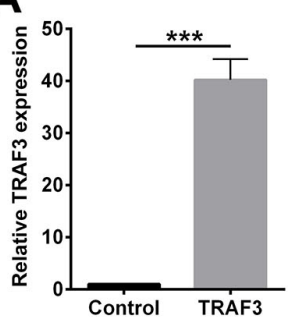

B

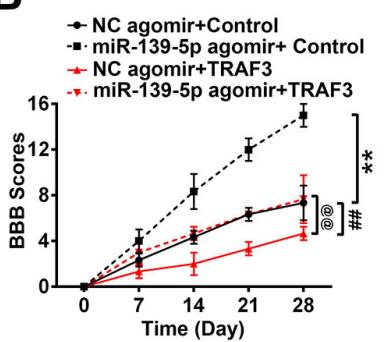

C
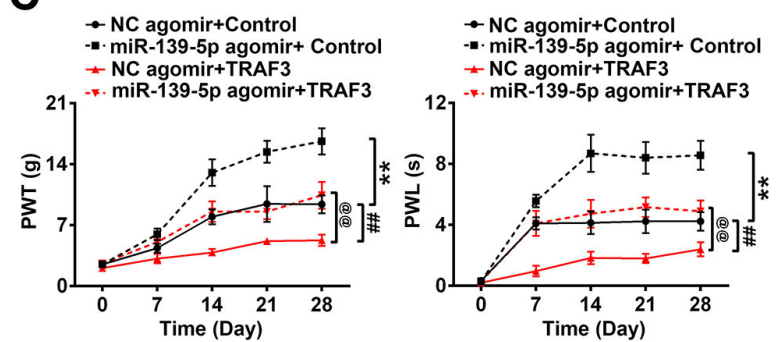

E
D

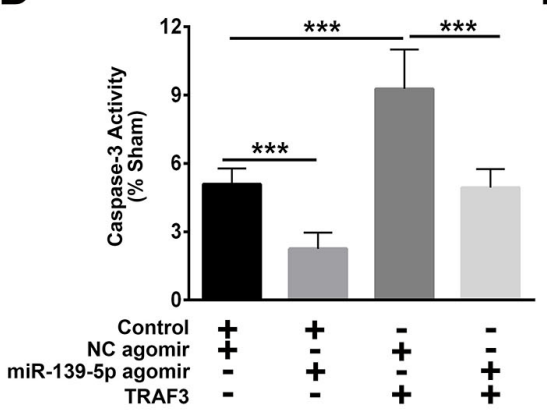

E Control

NC agomir
miR-139-5p agomir
TRAF3

TRAF3
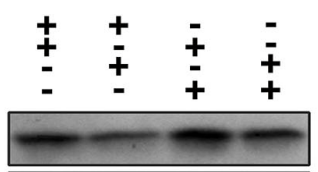

$\mathrm{BCI}$

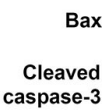

GAPDH

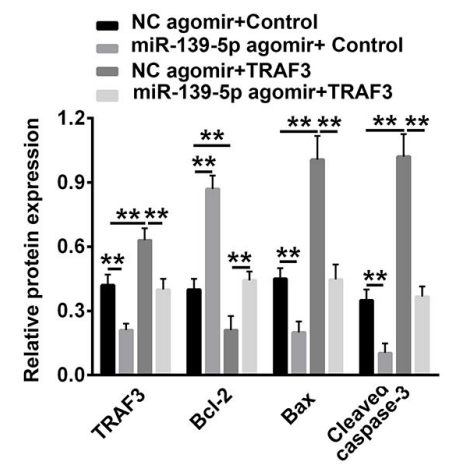

Figure 4. MiR-139-5p alleviates neural cell apoptosis by targeting TRAF3.

(A) TRAF3 overexpression in SCI rats. (B) The BBB scores at indicated time. (C) PWT and PWL were detected at 0, 7, 14, 21 and 28 days after SCI. (D) Caspase-3 activity in various groups. (E) TRAF3, BCl-2, Bax and Cleaved caspase-3 level. $N=6$, each group. ${ }^{*} P<0.01$, **P $<<0.01$ and ${ }^{* * *} \mathrm{P}<0.001$ refer to the group compared with the group of $\mathrm{NC}$ agomir + control. $\# p<0.01$ and \# \#p<0.01 refer to the group compared with the group of miR-139-5p agomir + control. 
scores indicated a significant increase in locomotor functional recovery by miR-139-5p overexpression. However, this effect was abolished by overexpression of TRAF3 (Fig. 4B). Furthermore, PWT and PWL were also significantly increased by overexpression of miR-139-5p by agomir and the effect was reversed by TRAF3 overexpression in the group of miR-139-5p agomir + TRAF3 (Fig. 4C). Caspase-3 activity was significantly decreased due to miR-139-5p overexpression whereas TRAF3 overexpression increased caspase-3 activity (Fig. 4D). Similarly, the effects of miR-139-5p on the TRAF3, Bcl-2, Bax and cleaved caspase- 3 expression were observed, indicating that TRAF3 acts as a mediator involved in the antiapoptosis effect of miR-139-5p in SCI rats (Fig. 4E). These results suggested that miR-139-5p reduced neural cell apoptosis by targeting TRAF3.

\section{DISCUSSION}

Spinal cord injury (SCI) is characterized by high disability and mortality due to the complicated nature of this disease despite various treatment strategies have been developed (Branco et al., 2007; Silva et al., 2014; Ahuja et al., 2017; Ghali \& Neuroscience 2017). Numerous evidences have suggested that the pathogenesis of SCI is associated with a variety of miRNAs including miR-137 (Gao et al., 2018), miR-221 (Zhao et al., 2018), miR-9 (Xu et al., 2016), and miR-136 (Jin et al., 2017). In our study, miR-139-5p was confirmed to be related to SCI with decreased expression in SCI rat model. MiR-139$5 \mathrm{p}$ overexpression improved locomotor functional recovery, attenuated allodynia and hyperalgesia and alleviated neural cell apoptosis in SCI rats. In addition, TRAF3 was shown to be a target of miR-139-5p by searching in the TargetScan 7.1 database and further studies verified that TRAF3 mediated the anti-apoptosis effect of miR$139-5 \mathrm{p}$ in SCI rats.

The role of miR-139-5p in the regulation of apoptosis in several cell types has been reported (Zhang et al., 2014; Huang et al., 2017). Other studies showed that miR-139-5p induced apoptosis in uterine leiomyoma cells (Chen et al., 2016), primary lung cancer cells (Sun et al., 2015), airway smooth muscle cells (Zhang et al., 2017) and diabetic rat pancreatic cells (Li et al., 2017). Therefore, miR-139-5p was considered as a pro-apoptotic factor in the pathogenesis of several diseases. However, emerging evidence reveals the role of miR-139-5p as a context-dependent regulatory factor which acts as either a pro-apoptotic or an anti-apoptotic factor. For instance, miR-139-5p was found to be an anti-apoptotic factor in hypoxia-ischemia brain damage by inhibiting the neural cell apoptosis (Qu et al., 2014). In our study, miR-139$5 p$ was also observed to have an anti-apoptotic effect on neural cell apoptosis in rats after SCI which further substantiate the anti-apoptotic role of miR-139-5p.

TNF receptor associated factor 3 (TRAF3) has been identified as a member of the TRAF family of intracellular signaling proteins (Saha \& Cheng 2006; He et al., 2007; Yi et al., 2014). Studies suggested that TRAF3 mediated apoptosis in several cell types. Increased expression of TRAF3 enhanced CD40 ligand (CD154)-induced human urothelial cell apoptosis by activating the c-Jun $\mathrm{N}$-terminal kinase (JNK)/activator protein-1 (AP-1) pathway (Georgopoulos et al., 2006). TRAF3 also plays the pro-apoptotic effect via phosphorylation of ASK-1 and MKK4 followed by JNK activation (Dunnill et al., 2017). TRAF3 serves as a target of miR-322 to enhance the high glucose-induced apoptosis of neural stem cells
(Gu et al., 2015). Furthermore, Ya Wu and others (Wu et al., 2014) established an acute SCI model and revealed that TRAF3 was remarkably up-regulated after SCI (Wu et al., 2014). In the present study, TRAF3 was found to be the target of miR-139-5p and functional studies suggested that TRAF3 mediated the anti-apoptotic effect of miR-139-5p in SCI rats.

Since accumulated evidences have revealed that the secondary injuries after SCI prolonged and complicated the clinical therapies (Oyinbo 2011), it is a promising strategy to focus on alleviating the secondary injuries such as cell apoptosis after SCI. In our study, the anti-apoptotic effect of miR-139-5p was verified in SCI rats. Furthermore, we found that the expression of miR$139-5 p$ was elevated in SCI rats. Meanwhile, our results suggested that upregulation of miR-139-5p could inhibit the expression of TRAF3, suggesting that miR-139-5p maybe a marker of apoptosis after SCI. Moreover, we verified that TRAF3 was a direct target of miR-139-5p through a constructed luciferase assay. Furthermore, we elucidated that miR-139-5p alleviated neural cell apoptosis by targeting TRAF3. Finally, by using a rat model, we demonstrated the protective effect of miR-139-5p on apoptosis after spinal cord injury. Our findings revealed that miR-139-5p inhibited apoptosis after spinal cord injury via the targeting of TRAF3. The outcomes of the present study might indicate a new therapeutic target for treatment of SCI. .

\section{Availability of data and materials}

All data generated or analyzed during this study are included in this published article.

\section{Authors' contributions}

ZYZ conceived and designed the experiments. LFS analyzed and interpreted the results of the experiments, YYY performed the experiments

\section{Conflict of interest}

The authors declare no conflict of interest.

\section{Ethics approval and consent to participate}

The experimental procedures and animal care were performed and approved by the Institutional Animal Care and Use Committee of The fifth affiliated hospital of Zhengzhou University (approval NO.2017Z086).

\section{Competing interests}

The authors state that there are no conflicts of interest to disclose.

\section{REFERENCES}

Abdullahi D, Annuar AA, Mohamad M, Aziz I, Rit JJ, Sanusi N (2016) Experimental spinal cord trauma: a review of mechanically induced spinal cord injury in rat models. Rev Neurosci 28: 15-20. https:// https://doi.org/10.1515/revneuro-2016-0050

Ahuja CS, Nori S, Tetreault L, Wilson J, Kwon B, Harrop J, Choi D, Fehlings MG (2017) Traumatic spinal cord injury-repair and regeneration. Neurosurgery 80: S9-S22. https://doi.org/10.1093/neuros/ nyw080

Ahuja CS, Wilson JR, Nori S, Kotter MRN, Druschel C, Curt A, Fehlings MG (2017) Traumatic spinal cord injury. Nat Rev Dis Primers 3: 17018. https://doi.org/10.1038/nrdp.2017.18

Anwar MA, Al Shehabi TS, Eid AH (2016) Inflammogenesis of secondary spinal cord injury. Front Cell Neurosic 10: 98. https://doi. org/10.3389/fncel.2016.00098 
Basso DM, Beattie MS, Bresnahan JC (1995) A sensitive and reliable locomotor rating scale for open field testing in rats. I Neurotrauma 12: 1-21. https://doi.org/10.1089/neu.1995.12.1

Bhimani AD, Kheirkhah P, Arnone GD, Nahhas CR, Kumar P, Wonais M, Hidrogo H, Aguilar E, Spalinski D (2017) Functional gait analysis in a spinal contusion rat model. Neurosci Biobehav Rev 83: 540-546. https://doi:10.1016/j.neubiorev.2017.09.007

Branco F, Cardenas DD, Svircev JN (2007) Spinal cord injury: a comprehensive review. Phys Med Rehabil Clin N Am 18: 651-679. https://doi.org/10.1016/j.pmr.2007.07.010

Chen H, Xu H, Meng YG, Zhang Y, Chen JY, Wei XN (2016) miR139-5p regulates proliferation, apoptosis, and cell cycle of uterine leiomyoma cells by targeting TPD52. Onco Targets Ther 9: 61516160. https://doi.org/10.2147/ott.s108890

Chivukula RR, Mendell JT (2008) Circular reasoning: microRNAs and cell-cycle control. Trends Biochem Sci 33: 474-481. https://doi. org/10.1016/j.tibs.2008.06.008

Choi DC, Lee JY, Lim EJ, Baik HH, Oh TH, Yune TY (2012) Inhibition of ROS-induced p38MAPK and ERK activation in microglia by acupuncture relieves neuropathic pain after spinal cord injury in rats. Exp Neurol 236: 268-282. https://doi.org/10.1016/j.expneurol.2012.05.014 236: 268-282.

Croce CM, Calin GA (2005) miRNAs, cancer, and stem cell division. Cell 122: 6-7. https://doi.org/10.1016/j.cell.2005.06.036

Diogo CC, da Costa LM, Pereira JE, Filipe V, Couto PA, Geuna S, Armada-da-Silva PA, Maurício AC, Varejão ASJN (2019) Kinematic and kinetic gait analysis to evaluate functional recovery in thoracic spinal cord injured rats. Neurosi Biobehav Rev 98: 18-28. https://doi. org/10.1016/i.neubiorev.2018.12.027

Dunnill CJ, Ibraheem K, Mohamed A, Southgate J, Georgopoulos NT (2017) A redox state-dictated signalling pathway deciphers the malignant cell specificity of CD40-mediated apoptosis. Oncogene 36: 2515-2528. https://doi.org/10.1038/onc.2016.401

Gao L, Dai C, Feng Z, Zhang L, Zhang Z (2018) MiR-137 inhibited inflammatory response and apoptosis after spinal cord injury via targeting of MK2. I Cell Biochem 119: 3280-3292. https://doi. org/10.1002/jcb.26489

Georgopoulos NT, Steele LP, Thomson MJ, Selby PJ, Southgate J Trejdosiewicz LK (2006) A novel mechanism of CD40-induced apoptosis of carcinoma cells involving TRAF3 and JNK/AP-1 activation. Cell Death Differ 13: 1789-1801. https://doi.org/10.1038/ si.cdd. 4401859

Ghali, MGZ (2017) Role of the medullary lateral tegmental field in sympathetic control. Neuroscience 16: 189-208. https://doi. org/10.3233/JIN-170010

Gu H, Yu J, Dong D, Zhou Q, Wang JY, Yang P (2015) The miR322-TRAF3 circuit mediates the pro-apoptotic effect of high glucose on neural stem cells. Toxicol Sci 144: 186-196. https://doi. org $/ 10.1093 /$ toxsci $/ \mathrm{kfu} 271$

Hama A, Sagen J (2009) Sustained antinociceptive effect of cannabinoid receptor agonist WIN 55,212-2 over time in rat model of neuropathic spinal cord injury pain. Development 46: 135.

He JQ, Oganesyan G, Saha SK, Zarnegar B, Cheng G (2007) TRAF3 and its biological function. Adv Exp Med Biol 597: 48-59. https:// doi.org/10.1007/978-0-387-70630-6_4

Huang LL, Huang LW, Wang L, Tong BD, Wei Q, Ding XS (2017) Potential role of miR-139-5p in cancer diagnosis, prognosis and therapy. Oncol Lett 14: 1215-1222. https://doi.org/10.3892/ ol.2017.6351

Hwang HW, Mendell JT (2007) MicroRNAs in cell proliferation, cell death, and tumorigenesis. Br J Cancer 96 (Suppl): R40-R44.

Jergova S, Gajavelli S, Pathak N, Sagen J (2016) Recombinant neural progenitor transplants in the spinal dorsal horn alleviate chronic central neuropathic pain. Pain 157: 977-989. https://doi. org/10.1097/j.pain.0000000000000471

Jin R, Xu S, Lin X, Shen M (2017) MiR-136 controls neurocytes apoptosis by regulating tissue inhibitor of metalloproteinases-3 in spinal cord ischemic injury. Biomed Pharmacother 94: 47-54. https://doi. org/10.1016/j.biopha.2017.07.053

Kadoya K, Lu P, Nguyen K, Lee-Kubli C, Kumamaru H, Yao L, Knackert J, Poplawski G, Dulin JN, Strobl H, Takashima Y, Biane
J, Conner J, Zhang SC (2016) Tuszynski MH Spinal cord reconstitution with homologous neural grafts enables robust corticospinal regeneration. Nat Med 22: 479-487. https://doi.org/10.1038/ nm.4066

Li J, Su L, Gong YY, Ding ML, Hong SB, Yu S, Xiao HP (2017) Downregulation of miR-139-5p contributes to the antiapoptotic effect of liraglutide on the diabetic rat pancreas and INS-1 cells by targeting IRS1. PLoS One 12: e0173576. https://doi.org/10.1371/ journal.pone.0173576

Ludwig PE, Patil AA, Chamczuk AJ, Agrawal DK (2017) Hormonal therapy in traumatic spinal cord injury. Am J Transl Res 9: 38813895.

Nakamura M, Okano H (2013) Cell transplantation therapies for spinal cord injury focusing on induced pluripotent stem cells. Cell Res 23: 70-80. https://doi.org/10.1038/cr.2012.171

Oyinbo CA (2011) Secondary injury mechanisms in traumatic spinal cord injury: a nugget of this multiply cascade. Acta Neurobiol Exp (Wars) 71: 281-299.

Qu Y, Wu J, Chen D, Zhao F, Liu J, Yang C, Wei D, Ferriero DM, Mu D (2014) MiR-139-5p inhibits HGTD-P and regulates neuronal apoptosis induced by hypoxia-ischemia in neonatal rats. Neurobiol Dis 63: 184-193. https://doi.org/10.1016/j.nbd.2013.11.023

Saha SK, Cheng G (2006) TRAF3: a new regulator of type I interferons. Cell Cycle 5: 804-807. https://doi.org/10.4161/cc.5.8.2637

Silva NA, Sousa N, Reis RL, Salgado AJ (2014) From basics to clinical: a comprehensive review on spinal cord injury. Prog Neurobiol 114: 25-57. https://doi.org/10.1016/j.pneurobio.2013.11.002

Sun C, Sang M, Li S, Sun X, Yang C, Xi Y, Wang L, Zhang F, Bi Y, Fu Y, Li D (2015) Hsa-miR-139-5p inhibits proliferation and causes apoptosis associated with down-regulation of c-Met. Oncotarget 6: 39756-39792. https://doi.org/10.18632/oncotarget.5476

Tabo E, Eisele JH Jr, Carstens E (1998) Force of limb withdrawals elicited by graded noxious heat compared with other behavioral measures of carrageenan-induced hyperalgesia and allodynia. J Neurosci Methods 81: 139-149. https://doi.org/10.1016/s01650270(98)00018-1

Varma AK, Das A, Wallace Gt, Barry J, Vertegel AA, Ray SK, Banik NL (2013) Spinal cord injury: a review of current therapy, future treatments, and basic science frontiers. Neurochem Res 38: 895-905. https://doi.org/10.1007/s11064-013-0991-6

Wu Y, Zheng M, Wang S, Song C, Wang C, Xiao Y, Xu L, Xu X (2014) Spatiotemporal pattern of TRAF3 expression after rat spinal cord injury. I Mol Histol 45: 541-553. https://doi.org/10.1007/ s10735-014-9575-2

Xu Y, An BY, Xi XB, Li ZW, Li FY (2016) MicroRNA-9 controls apoptosis of neurons by targeting monocyte chemotactic proteininduced protein 1 expression in rat acute spinal cord injury model. Brain Res Bull 121: 233-240. https://doi.org/10.1016/j.brainresbull.2016.01.011

Yaksh TL, Rudy TA (1976) Chronic catheterization of the spinal subarachnoid space. Behavior 17: 1031-1036.

Yi Z, Lin WW, Stunz LL, Bishop GA (2014) Roles for TNF-receptor associated factor 3 (TRAF3) in lymphocyte functions. Cytokine Growth Factor Rev 25: 147-156. https://doi.org/10.1016/j.cytogfr. 2013.12.002

Zhang H, Sun Z, Yu L, Sun J (2017) MiR-139-5p inhibits proliferation and promoted apoptosis of human airway smooth muscle cells by downregulating the Brg1 gene. Respir Physiol Neurobiol 246: 9-16. https://doi.org/10.1016/j.resp.2017.07.004

Zhang L, Dong Y, Zhu N, Tsoi H, Zhao Z, Wu CW, Wang K, Zheng S, Ng SS, Chan FK, Sung JJ, Yu J (2014) microRNA-139-5p exerts tumor suppressor function by targeting NOTCH1 in colorectal cancer. Mol Cancer 13: 124. https://doi.org/10.1186/1476-4598-13-124

Zhao D, Deng SC, Ma Y, Hao YH, Jia ZH (2018) miR-221 alleviates the inflammatory response and cell apoptosis of neuronal cell through targeting TNFAIP2 in spinal cord ischemiareperfusion. Neuroreport 29: 655-660. https://doi.org/10.1097/ wnr.0000000000001013 